nephron

Practice
Nephron 2015;131:237-241

DOI: $10.1159 / 000441426$
Received: April 21, 2015

Accepted after revision: September 30, 2015

Published online: November 11, 2015

\title{
Can Acute Kidney Injury Be Considered a Clinical Quality Measure?
}

\author{
Matthew T. James ${ }^{\mathrm{a}}$ Neesh Pannu ${ }^{\mathrm{b}}$ \\ ${ }^{a}$ Department of Medicine and Community Health Sciences, Cumming School of Medicine, University of Calgary, \\ Calgary, ${ }^{b}$ Department of Medicine, Faculty of Medicine, University of Alberta, Edmonton, Alta., Canada
}

\section{Key Words}

Acute kidney injury - Performance measurement .

Clinical quality improvement

\begin{abstract}
Quality indicators are measurements of healthcare outcome, process, or structure that can be used as tools to measure the quality of care and identify opportunities for improvement. Acute kidney injury (AKI) has many characteristics that make it a potential target for quality indicator development. It is common, associated with a high risk of adverse outcomes, and there are reports of gaps in the quality of care in several clinical settings despite publication of evidence-based guidelines. Substantial work has already been undertaken to develop quality measures related to AKI following percutaneous coronary interventions and major surgical procedures. This paper reviews the current literature that has addressed issues of prevention or management of AKI as outcome, process, or structure quality indicators in these clinical settings. Several current controversies about the appropriateness of such indicators related to AKI are identified. Further research to strengthen the evidence-base supporting prevention and management initiatives for AKI across all relevant clinical settings is needed to clarify the role of AKI as a target for clinical quality indicators.

(c) 2015 S. Karger AG, Basel
\end{abstract}

\section{Introduction}

Healthcare organizations and professional societies are increasingly recognizing the importance of improving the quality of healthcare. The development of quality indicators (also known as quality measures or performance indicators) is one approach to quality improvement. Quality indicators are measurements of healthcare outcome, process, or structure that provide practitioners and institutions that deliver health services with tools to measure the quality of their care and identify opportunities for improvement.

Quality indicators may address one or more domain of quality, including timeliness, safety, effectiveness, efficiency, equity, and patient-centeredness. Issues of feasibility should be taken into account in the development of quality indicators, given the additional burden required to measure and report on an indicator. Implementation of any potential quality indicator may also pose unintended consequences, which must be carefully considered. Sophisticated methods have been developed to de-

Contribution from the AKI and CRRT 2015 Symposium at the 20th International Conference on Advances in Critical Care Nephrology, Manchester Grand Hyatt, San Diego, Calif., USA, February 17-20, 2015.

\section{KARGER 125}

(๑) 2015 S. Karger AG, Basel

$1660-8151 / 15 / 1314-0237 \$ 39.50 / 0$ 
rive quality indicators to ensure that these measures are clinically relevant, aligned with best evidence, and are feasible to implement $[1,2]$.

Acute kidney injury (AKI) has many characteristics that make it a potential target for quality indicator development. AKI is common and associated with a high risk of adverse outcomes, yet potentially preventable or treatable with appropriate interventions. Recently, evidencebased clinical practice guidelines for AKI prevention, recognition, and management have been published [3]. However, there is wide variability in care and outcomes of AKI, including important evidence-care gaps for AKI described in several clinical settings [4]. This paper reviews the characteristics of quality indicators, and evaluates the current body of literature that has addressed the validity of $\mathrm{AKI}$ as a target of quality indicators for percutaneous coronary interventions (PCI) and major surgery.

\section{Characteristics of Quality Indicators}

The development of quality indicators is first informed by high-quality evidence about care (table 1). Systematic reviews and clinical practice guidelines provide an important evidence-base for developing quality indicators. Information on the clinical epidemiology of the condition of interest, variability in care practices, and evidencepractice gaps are also relevant to ensure quality indicators target areas of care where there are meaningful opportunities for improvement. Quality indicators are also informed by the opinions and experience of clinicians with knowledge in the area, and peer review and public comment is often also incorporated to refine the selection of quality indicators.

Process indicators focus on measures of the care delivered to patients, including the services and treatments they receive. They have the advantage of being directly under the control of care providers, and thus can be more easily targeted for change. Processes indicators are most valuable when robust evidence demonstrates that they directly lead to improved clinical outcome, and when true evidence-care gaps in care are known to exist. Examples of potential process indicators for AKI could include risk stratification for AKI in specific clinical scenarios, collection of urine output or serum creatinine measurements to detect AKI, and use of AKI prevention strategies.

Outcome indicators measure the impact of care on the health status of patients and populations. They focus on the results of care that are most important to clinicians and patients. Outcome indicators depend upon valid risk- adjustment methods to enhance the comparability of outcomes across different delivery teams, care settings, and systems. Although these indicators are attractive, they can be less meaningful when there are prominent influences on clinical outcomes that are beyond the control of care providers or the health system. Examples of outcome indicators for AKI could include the incidence of AKI following radiocontrast procedures or major surgery.

Structural indicators relate to the physical and organizational characteristics within which healthcare occurs. These indicators have the advantage of being easier to measure because they can be captured at the level of health organizations and do not require sophisticated risk adjustment methods. Structural indicators for AKI might include an institution's adoption of standardized contrast-induced AKI (CI-AKI) prophylaxis protocols for patients undergoing radiocontrast procedures or adherence to protocol-based management of patients with hemodynamic instability to prevent development or progression of AKI.

\section{Quality Indicator Development for AKI Following Percutaneous Coronary Procedures}

Substantial work has already been undertaken by organizations in Canada and the United States to develop quality measures related to CI-AKI following PCI. In Canada, quality indicators for PCI were developed by a national expert panel in 2008 [5]. Potential indicators were identified from published guidelines, randomized trials, and outcomes studies. A modified Delphi process was used to rate potential indicators, and a panel meeting was held to obtain consensus. The final set of quality indicators included (i) a structural indicator that a standardized protocol be in place to minimize CI-AKI, (ii) a process indicator for renal function to be assessed before the procedure, and (iii) an outcome indicator for renal failure requiring hemodialysis after percutaneous intervention.

A recent observational study from 13 hospitals that perform PCI in the province of Ontario, Canada reported high levels of adherence to the quality indicator for assessment of renal function before PCI between 2009 and 2010 , observed in $94.5 \%$ of cases, ranging from 84.0 to $100 \%$ of cases across the hospitals studied [6]. Renal failure requiring dialysis was a rare outcome after PCI, observed in $0.2 \%$ of patients (range $0-1.0 \%$ across the hospitals studied). No information was provided on the catheterization lab adherence to the structural indicator for 
Table 1. Characteristics of quality indicators

\begin{tabular}{ll}
\hline Characteristic & Features \\
\hline Evidence base & $\begin{array}{l}\text { Structural indicators: closely related to a clinically important process of care that is in turn related to } \\
\text { an important clinical outcome } \\
\text { Process indicators: supported by empirical evidence relating a process of care to an important } \\
\text { clinical outcome } \\
\text { Outcome indicators: clinically important and adjusted for relevant clinical characteristics using } \\
\text { appropriate methods }\end{array}$ \\
$\begin{array}{l}\text { Definition: the numerator (e.g. event, process, structure) and denominator (e.g. patient group, } \\
\text { hospital) required to calculate the indicator are clearly defined and clinically meaningful } \\
\text { Reliability: the indicator is reproducible across care settings } \\
\text { Face validity: the indicator appears to measure what is intended } \\
\text { Content validity: the indicator captures most meaningful elements of care } \\
\text { Construct validity: the indicator correlates with other measures of the same aspect of care }\end{array}$ \\
$\begin{array}{l}\text { Data required for measurement can be obtained with reasonable effort and cost in the required } \\
\text { time-frame }\end{array}$ \\
$\begin{array}{l}\text { Actionable: those accountable can influence the structure of care, process, or outcome } \\
\text { Risk of harm: the risk of undesirable, unintended consequences is low }\end{array}$ \\
\hline Accountability
\end{tabular}

use of standardized protocols for CI-AKI prevention. While these findings provide encouraging information about the quality of care for AKI in the setting of PCI in Canada, information is lacking as to whether the use of these quality indicators or related quality improvement initiatives have been associated with improved patient outcomes.

Recently, a set of performance measures were published for adults undergoing PCI in the United States, endorsed by several American cardiology organizations [7]. A number of quality indicators related to CI-AKI after PCI were considered in the development of these measures. The first indicator selected was a process indicator for assessment of estimated glomerular filtration (eGFR) rate as a standard part of the pre-procedural work-up of patients undergoing coronary angiography and intervention. The work group based its selection as an indicator in large part because kidney function was deemed important for dosing of contrast media, and because current guidelines recommend that patients with reduced eGFR receive hydration prior to the procedure to prevent CIAKI. The second indicator selected by the work group was documentation of the total volume of contrast administered to a patient. The work group cited the relationship between increasing the volume of contrast administrated and the risk of CI-AKI as the main rationale for selection of this process indicator.
The US workgroup also considered several potential indicators related to CI-AKI that were not included in the final set of quality indicators. A measure of the incidence of dialysis after PCI was considered; however, this was not included because the work group felt that unexpected dialysis after PCI was rare, and usually occurred among patients who already had advanced kidney disease before the PCI. They cited the concern that this measure could result in unintended consequences by dissuading care providers from offering PCI to patients with chronic kidney disease, who are very high risk for coronary artery disease and adverse outcomes yet are already less likely to receive angiography and PCI. Although several members of the work group supported a measure of CI-AKI based on changes in serum creatinine alone, the work group refrained from selecting this as an outcome measure, citing controversy about the diagnosis of CI$\mathrm{AKI}$ and the concern that it would lead to unnecessary blood tests. There is a growing body of literature suggesting that contemporary definitions of AKI based on small changes in kidney function (e.g. KDIGO AKI stage 1) may be representative of biological and laboratory variation rather than AKI, particularly when relative changes are used in patients with low baseline serum creatinine, or when absolute increases are used in patients with high baseline creatinine [8-10]. Although these criteria may be useful for early identification of patients at risk of AKI, 
they are undesirable features of current AKI criteria for development of AKI as a clinically relevant outcome measure. AKI defined by larger serum creatinine changes (e.g. KDIOG AKI stage 2 or 3 ) or AKI that contributes to a prolonged stay in hospital, leads to a hospital readmission, requires dialysis treatment, or is listed as a cause of death may constitute a more appropriate outcome indicator for AKI.

Finally, the work group also considered a structural quality indicator of a protocol for prevention of CI-AKI; however, it decided that the existing evidence base was not strong enough to support inclusion of such an indicator. The work group suggested that process-based measures for reporting pre-procedural eGFR and contrast dose should be sufficient to capture important data to guide local improvement efforts for CI-AKI, and that consideration for inclusion of structural indicators for CI-AKI prevention protocols might be warranted in the future if further favorable evidence accumulates.

Observational studies from the United States provide encouraging signs that there may be value to efforts to report CI-AKI after PCI and to develop quality improvement strategies for prevention of CI-AKI. In a cohort study of over 30,000 patients with myocardial infarction from 56 US hospitals between 2000 and 2008, Amin et al. [11] reported that the incidence of AKI varied significantly between hospitals, with $26 \%$ of the risk of developing AKI attributable to differences between hospitals after multivariable adjustment. Importantly, there was a significant decline in the incidence of AKI among patients who underwent invasive cardiac procedures (from $24.6 \%$ in 2000 to $16.5 \%$ in 2008 ) despite an increase in the age and prevalence of AKI risk factors over this time period. The authors hypothesized that greater clinician awareness, better risk stratification, or increased use of AKI prevention efforts may underlie this finding. Further, a prospective multicenter quality improvement study to prevent CI-AKI in patients undergoing PCI in 6 hospitals in New England between 2007 and 2012, reported a significant $21 \%$ reduction in the adjusted rate of CI-AKI associated with this initiative [12]. This quality improvement project focused on team-based interventions including standardizing hydration orders, reducing NPO time, mandating a fluid bolus before the procedure, limiting contrast volume, and patient education about selfhydration using oral fluids. These results suggest that quality improvement interventions for CI-AKI can substantially reduce the incidence of AKI, and highlight the potential role of AKI as a quality indicator in PCI. However, future studies for CI-AKI quality improvement ini- tiative remain necessary, particularly studies to examine whether targeting CI-AKI prevention influences hard clinical and economic endpoints, such as the length of days patients stay in hospital, rates of dialysis, and downstream complications such as CKD, cardiovascular events, and end-stage renal disease.

\section{Quality Indicator Development for AKI Following Major Surgery}

Efforts have also been made to develop AKI as a quality indicator following major surgery. The Agency for Healthcare Research and Quality in the United States developed a Patient Safety Indicator (PSI) 10 'Postoperative Physiologic and Metabolic Derangement', which uses administrative data to detect postoperative AKI requiring dialysis as well as diabetes-related complications [13]. Over $90 \%$ of events identified by this PSI represent episodes of AKI and validation studies using administrative data sources have suggested moderate validity [14]. Misclassification of diabetes complications and false positive results due to preoperative renal failure requiring dialysis appear to underlie limitations in the sensitivity and positive predictive value of this indicator. The use of codes to distinguish patients requiring dialysis at the time of hospital admission from those who develop requirements for dialysis following admission to hospital, along with removal of the diabetes criteria, has been suggested to improve the performance of this indicator and provide a more useful outcome measure for AKI after surgery.

Most recently, the National Quality Forum included risk-adjusted postoperative renal failure as one of 21 quality measures developed for surgery [15]. This indicator, endorsed by the Society of Thoracic Surgeons, measures the proportion of adults without preexisting kidney failure undergoing isolated coronary artery bypass grafting who develop postoperative renal failure or require dialysis.

We recently conducted a modified Delphi process in Alberta, Canada to identify indicators of high quality processes of care related to AKI prevention, identification, and management after major surgery [16]. Consensus was obtained on 16 indicators with high perceived validity; however, our study did not address the feasibility of measuring these indicators. At present, there is lack of literature on risk adjusted measurement of postoperative AKI or evaluation of these processes of care indicators for AKI across institutions. Further research will be required to validate proposed quality indicators for AKI after ma- 
jor surgery, and to evaluate whether they can be effectively employed to measure and improve the quality of care for AKI after major surgery.

\section{Conclusion and Future Directions}

Substantial work has already been done to develop potential quality indicators for AKI following PCI and major surgery. Measurement of pre-procedure kidney function and the volume of contrast dye administered have achieved some consensus as AKI quality indicators in PCI in both Canada and the United States. However, controversy remains about the appropriateness of AKI requiring dialysis as an outcome indicator and use of standardized protocols for CI-AKI prevention as a structural indicator. Further, concerns about misclassification continue to evoke controversy about whether current AKI criteria defined by small changes in serum creatinine alone are appropriate for use as a valid outcome indicator. Further research is needed to strengthen the evidence-base supporting AKI prevention and management approaches across all clinical settings. Future work will also need to carefully evaluate whether quality improvement strategies based on current best practices for AKI prevention and care can achieve desired outcomes without unintended consequences. Given the adverse health outcomes and costs associated with AKI, further research investments to clarify the role of AKI as a quality indicator appear warranted.

\section{References}

1 Boulkedid R, Abdoul H, Loustau M, Sibony O, Alberti C: Using and reporting the Delphi method for selecting healthcare quality indicators: a systematic review. PLoS One 2011; 6:e20476.

-2 Normand SL, McNeil BJ, Peterson LE, et al: Eliciting expert opinion using the Delphi technique: identifying performance indicators for cardiovascular disease. Int J Qual Health Care 1998;10:247-260.

3 Kidney Disease: Improving Global Outcomes (KDIGO). Clinical practice guideline for acute kidney injury. Kidney Int Suppl 2012;2: $1-138$.

4 National Confidential Enquiry into Patient Outcome and Death (NCEPOD). Adding Insult to Injury. London, NCEPOD, 2009.

5 Ko DT, Wijeysundera HC, Zhu X, Richards J, Tu JV; National Expert Panel: Canadian quality indicators for percutaneous coronary interventions. Can J Cardiol 2008;24:899-903.

6 Czarnecki A, Prasad TJ, Wang J, Wijeysundera HC, Cheema AN, Džavík V, Natarajan MK, Simpson CS, So DY, Syed J, Tu JV, Ko DT: Adherence to process of care quality indicators after percutaneous coronary intervention in Ontario, Canada: a retrospective observational cohort study. Open Heart 2015; 2:e000200.

7 Nallamothu BK, Tommaso CL, Anderson HV, Anderson JL, Cleveland JC Jr, Dudley RA, Duffy PL, Faxon DP, Gurm HS, Hamilton LA, Jensen NC, Josephson RA, Malenka
DJ, Maniu CV, McCabe KW, Mortimer JD, Patel MR, Persell SD, Rumsfeld JS, Shunk KA, Smith SC Jr, Stanko SJ, Watts B: ACC/AHA/ SCAI/AMA-convened PCPI/NCQA 2013 performance measures for adults undergoing percutaneous coronary intervention: a report of the American College of Cardiology/ American Heart Association Task Force on Performance Measures, the Society for Cardiovascular Angiography and Interventions, the American Medical Association-Convened Physician Consortium for Performance Improvement, and the National Committee for Quality Assurance. J Am Coll Cardiol 2014;63:722-745.

-8 Zeng X, McMahon GM, Brunelli SM, Bates DW, Waikar SS: Incidence, outcomes, and comparisons across definitions of AKI in hospitalized individuals. Clin J Am Soc Nephrol 2014;9:12-20.

-9 Sileanu FE, Murugan R, Lucko N, Clermont G, Kane-Gill SL, Handler SM, Kellum JA: AKI in low-risk versus high-risk patients in intensive care. Clin J Am Soc Nephrol 2015;10: 187-196.

10 Lin J, Fernandez H, Shashaty MG, Negoianu D, Testani JM, Berns JS, Parikh CR, Wilson FP: False-positive rate of AKI using consensus creatinine-based criteria. Clin J Am Soc Nephrol 2015;10:1723-1731.

11 Amin A, Salisbury A, McCullough PA, Gosch K, Spertus JA, Venkitachalam L, Stolker JM, Parikh CR, Masoudi FA, Jones PG, Kosiborod
M: Trends in the incidence of acute kidney injury in patients hospitalized with acute myocardial infarction. Arch Intern Med 2012; 172:246-253.

12 Brown JR, Solomon RJ, Sarnak MJ, McCullough PA, Splaine ME, Davies L, Ross CS, Dauerman HL, Stender JL, Conley SM, Robb JF, Chaisson K, Boss R, Lambert P, Goldberg DJ, Lucier D, Fedele FA, Kellett MA, Horton S, Phillips WJ, Downs C, Wiseman A, MacKenzie TA, Malenka DJ; Northern New England Cardiovascular Disease Study Group: Reducing contrast-induced acute kidney injury using a regional multicenter quality improvement intervention. Circ Cardiovasc Qual Outcomes 2014;7:693-700.

13 http://www.qualityindicators.ahrq.gov/ modules/psi_overview.aspx.

14 Zrelak PA, Romano PS, Tancredi DJ, Geppert JJ, Utter GH: Validity of the AHRQ patient safety indicator for postoperative physiologic and metabolic derangement based on a national sample of medical records. Med Care 2013;51:806-811.

15 http://www.qualityforum.org/Home.aspx.

16 James MT, Pannu N, Barry R, Karsanji D, Tonelli M, Hemmelgarn BR, Manns BJ, Bagshaw SM, Stelfox HT, Dixon E: A modified Delphi process to identify process of care indicators for the identification, prevention and management of acute kidney injury after major surgery. Can J Kidney Health Dis 2015;2: 11. 